\title{
Intervención educativa para la evaluación de la simetría nasal en pacientes operados de labio y paladar hendido
}

\author{
Tatiana I. Castillo-Torres ${ }^{1 *}$, María L. Peralta-Pedrero², Marcia R. Pérez-Dosal ${ }^{3}$, \\ Norma M. Dominguez-Martínez ${ }^{4}$, Olga Apodaca-García ${ }^{5}$ y Ariana Cruz-Sandoval ${ }^{1}$ \\ ${ }^{1}$ Departamento de Ortodoncia, Fundación de Anomalías Craneofaciales Fernando Ortiz Monasterio, Ciudad de México, México; ${ }^{2}$ Universidad \\ Nacional Autónoma de México, Ciudad de México, México; ${ }^{3}$ Departamento de Cirugía Plástica y Reconstructiva, Instituto Nacional de Pediatría, \\ Ciudad de México, México; ${ }^{4}$ Smile Train Proyecto América Central y Caribe, New York, USA; ${ }^{5}$ Centro de Especialidades Odontológicas Dr. Renato \\ de Toledo Leonardo, Tijuana, Baja California, México
}

\begin{abstract}
Resumen
Introducción: La asimetría nasal es una de las características más frecuentes de los pacientes con labio y paladar hendido; la medición antropométrica (MA) es un método accesible y confiable que puede ser utilizado por los especialistas para evaluarlo. El objetivo de este estudio fue evaluar la efectividad de una intervención educativa aplicada a especialistas que tratan pacientes con labio y paladar hendido para incrementar la precisión en las MA en la evaluación de la asimetría nasal. Métodos: Estudio cuasiexperimental. Resultados: En la primera fase del estudio, 5 de las 13 medidas antropométricas reportaron una diferencia de medias $(D M)>1.5 \mathrm{~mm}$, y 12 de las 13 MA mostraron resultados mayores a dos desviaciones estándar (DE). Los resultados en la segunda fase evidenciaron 11 de las 13 MA con DM < 1 mm, y 9 de las 13 MA fueron menores a dos DE. Conclusiones: La intervención realizada con el Manual de medidas antropométricas aumentó los conocimientos de los especialistas sobre la anatomía, puntos y MA que se pueden utilizar para evaluar la asimetría nasal en pacientes con labio y paladar hendido. La intervención educativa ayuda a aumentar el acuerdo entre los evaluadores para realizar una evaluación confiable de la asimetría nasal en pacientes con estos padecimientos.
\end{abstract}

Palabras clave: Intervención educativa. Antropometría. Labio y paladar hendido. Asimetría nasal.

\section{Educational intervention for the evaluation of nasal symmetry in cleft lip and palate operated patients}

\begin{abstract}
Background: Nasal asymmetry is one of the most frequent characteristics of patients with cleft lip and palate. The anthropometric measurement (AM) is an accessible and reliable method that can be used by specialists to evaluate nasal asymmetry in patients with cleft lip and palate. The aim of this study was to evaluate the effectiveness of an educational intervention applied to cleft lip and palate specialists to increase the accuracy of AM in the evaluation of nasal asymmetry. Methods: Quasi-experimental study. Results: In the first phase of the study, five of the 13 AM reported a mean difference $(M D)>1.5 \mathrm{~mm}$, and 12 of the $13 \mathrm{AM}$ showed results greater than $2 \mathrm{SD}$ (standard deviations). In the second phase, the results
\end{abstract}

Correspondencia:

*Tatiana Izchel Castillo-Torres

E-mail: izchelct@gmail.com
Disponible en internet: 23-03-2020 Bol Med Hosp Infant Mex.2020;77(2):68-75 www.bmhim.com 1665-1146/C 2019 Hospital Infantil de México Federico Gómez. Publicado por Permanyer. Éste es un artículo open access bajo la licencia CC BY-NC-ND (http://creativecommons.org/licenses/by-nc-nd/4.0/). 
showed 11 of the $13 A M$ with $M D<1 \mathrm{~mm}$, and 9 of the 13 AM were less than 2 SD. Conclusions: The intervention carried out with the manual of AMs increased the knowledge of the specialists on the anatomy points and AM that can be used to evaluate the nasal asymmetry in patients with cleft lip and palate. Educational intervention help to increase the agreement between examiners to perform a reliable evaluation of nasal asymmetry in patients with these conditions.

Key words: Educational needs. Anthropometry. Cleft lip and palate. Nasal asymmetry.

\section{Introducción}

El labio hendido con o sin paladar hendido es una de las alteraciones craneofaciales más frecuentes que resultan de una falta de unión de los procesos frontonasales con los procesos maxilares en las primeras semanas de vida intrauterina'. El labio y paladar hendido (LPH) se presenta con mayor frecuencia en asiáticos y con menor frecuencia en africanos. México se encuentra en el segundo lugar a escala mundial de prevalencia de labio hendido con o sin paladar hendido ${ }^{2}$. Se ha calculado que 1 de cada 358 a 500 recién nacidos vivos presentan labio con 0 sin paladar hendido ${ }^{3}$.

Los pacientes con LPH presentan diferentes secuelas, como deformidad nasal y alteración en el crecimiento craneofacial ${ }^{4}$. La deformidad nasal primaria se presenta antes de la primera cirugía, y se debe a la disrupción que la fisura provoca en las estructuras anatómicas, por lo que se produce la discontinuidad de la fosa piriforme, la inserción anómala de las fibras musculares, el desplazamiento del cartílago nasal, la concavidad del domo nasal, la disminución en la longitud de la columnela y el aumento en la longitud horizontal de la fosa nasal y piso nasal ${ }^{5-7}$. La deformidad nasal secundaria se presenta después de la cirugía, entre cuyas causas se encuentra la deficiente técnica quirúrgica, el crecimiento del septum nasal en etapas de desarrollo y, una de las más importantes, la deformidad nasal primaria con la que nació el paciente ${ }^{5}$.

Un estudio realizado por Puente, et al. con fotogramametría mostró que la deformidad nasal posterior a la cirugía se presenta en el $70 \%$ de los casos de cierre labial con corrección nasal primara ${ }^{8}$. El registro fotográfico de la deformidad nasal puede ser de gran ayuda; sin embargo, este registro puede resultar sesgado si no se estandariza la técnica de los evaluadores y el método de toma de las fotografías ${ }^{5}$.

La evaluación subjetiva causa variación de los datos con base en la experiencia del evaluador. En la actualidad, se busca realizar mediciones objetivas para mejorar su validez ${ }^{5}$. La antropometría es una técnica que se encarga de realizar mediciones que proporcionen datos cuantitativos para determinar objetivamente la anatomía, realizar diagnósticos, plan de tratamientos o evaluar resultados ${ }^{9}$.

La antropometría se clasifica en directa, dentro de la que se encuentra la clínica, la cual es considerada como el «estándar de oro», e indirecta, que comprende los modelos de estudio, fotografía y tomografía ${ }^{9,10}$. Las medidas antropométricas (MA) se han utilizado para la evaluación nasal y facial; sin embargo, para la confiabilidad de los datos, se requiere del entrenamiento de los evaluadores s,9-11. $^{\text {. }}$

La confiabilidad evalúa la estabilidad de la información; es decir, que la información obtenida sea similar cuando un procedimiento de medición es realizado más de una vez ${ }^{12,13}$. La confiabilidad de una medición evalúa el acuerdo intra e interobservador. Si los distintos examinadores discrepan en la evaluación, la MA tendrá poca utilidad clínica al no concordar en la lectura y presentación de los datos.

Para evaluar la confiabilidad de una medida se deben de estudiar diferentes aspectos, como los que se describen a continuación:

- Repetibilidad de la medida. Para evaluarla, se aplica el método de medir de la misma manera y a los mismos sujetos en dos o más tiempos (prueba-reprueba o test-retest). Una medida reproducible debe arrojar el mismo resultado antes de compararse con otro método ${ }^{13}$.

- Concordancia intraobservador e interobservador. Representa el grado de consistencia para leer o interpretar un resultado de un observador consigo mismo y entre otros observadores. Las dos medidas que se realizan deben ser independientes una de otra ${ }^{12,13}$.

- Consistencia interna. Se refiere a la homogeneidad de los ítems que miden un mismo atributo ${ }^{12,13}$.

Múltiples estudios proponen la evaluación de los resultados quirúrgicos de la forma nasal; sin embargo, no todos los cuidadores de la salud conocen las herramientas necesarias y la forma de realizar MA en pacientes operados de LPH. El objetivo de esta investigación fue probar la efectividad de una intervención educativa aplicada a cuidadores de la salud de pacientes con LPH, para incrementar la precisión de las MA necesarias y realizar mediciones objetivas y cuantitativas para un diagnóstico de asimetría nasal. 


\section{Métodos}

El estudio se realizó del 20 al 23 de octubre del 2017 en el XVI Congreso de la Asociación Mexicana de Labio, Paladar Hendido y Anomalías Craneofaciales, A.C. mediante un diseño cuasiexperimental de antes y después.

Se reclutaron dos grupos de asistentes que fueran cuidadores de la salud, que colaboraran en una clínica multidisciplinaria de LPH con manejo de tratamiento de la deformidad nasal para realizar conformadores nasales o para planear cirugías de corrección nasal secundaria. Estos cuidadores de la salud debían contar con un mínimo de experiencia de 3 años y asistir al taller con un vernier metálico con pantalla digital, con tolerancia máxima de error en la medición de $0.05 \mathrm{~mm}$.

Para el estudio, se preparó un manual que esquematizó las MA que evalúan la simetría nasal. El estudio cuasiexperimental se desarrolló en tres fases.

\section{Preparación de manual}

Búsqueda bibliográfica, recuperación de documento y análisis del material para la identificación de las MA que miden la deformidad nasal en pacientes con LPH. La planeación del taller incluyó el manual, cuestionarios de evaluación (pre y pos) y, además, a cada asistente se le entregó un par de modelos de yeso tomados de la nariz de un paciente con LPH, con el objetivo de cegar a los asistentes durante la evaluación test-retest. Cada par de modelos de yeso era de diferente color: modelo 1 (morado) y modelo 2 (blanco) (Fig. 1).

\section{FASE 1}

Se entregaron los dos modelos de yeso y se otorgaron 40 min para esta fase. Los asistentes realizaron las MA solicitadas en el cuestionario preintervención en el modelo 1 (morado). Una vez concluida la evaluación, se les proporcionó el modelo 2 (blanco), y se les solicitó que realizaran y registraran la evaluación correspondiente. Los asistentes al taller desconocían que ambos modelos de yeso eran del mismo paciente; además, no se permitió que consultaran las respuestas previas.

\section{FASE 2}

Intervención educativa. Se otorgaron 60 min para esta fase. Se llevó a cabo la presentación teórica-interactiva del Manual de medidas antropométricas para la

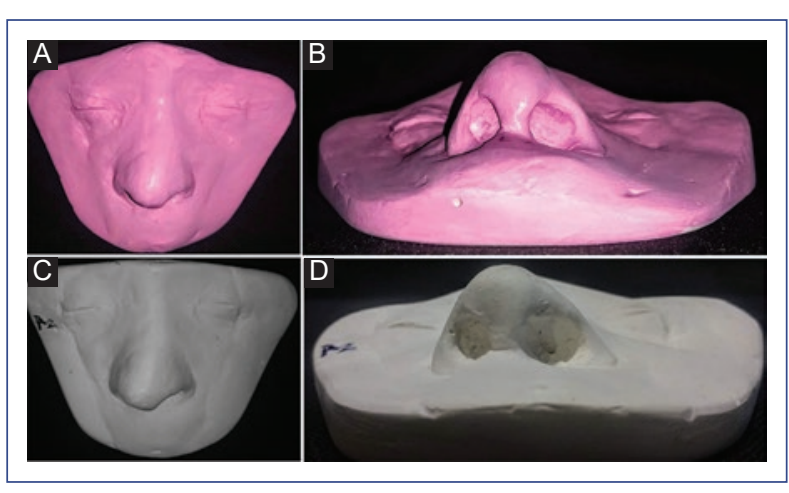

Figura 1. Vista frontal (A) y vista basal (B) del primer modelo de yeso (color morado). Vista frontal (C) y vista basal (D) del segundo modelo de yeso (color blanco).

evaluación de la simetría nasal, realizada en tres apartados:

a)Localización y definición de puntos antropométricos. Con apoyo de una presentación, se explicó teóricamente y se presentó de manera visual e interactiva la localización de los puntos antropométricos en la nariz de una paciente con LPH.

b) Procedimiento para la medición de los parámetros antropométricos para la evaluación clínica de la simetría nasal en pacientes con LPH. Se presentó el instrumento de medición vernier: el encendido, las partes que lo componen, el sistema métrico para registrar las medidas y la forma de usarlo para realizar las mediciones con antropometría directa, en tejidos de los pacientes, o indirecta, en modelos de yeso. Después de la exposición teórica y la presentación visual -interactiva de los esquemas de las MA-, se entregó material impreso a los asistentes al taller para marcar los puntos antropométricos y consultar si era adecuada su localización.

c) Dudas y preguntas. Por último, se les otorgaron $30 \mathrm{~min}$ para realizar preguntas y esclarecer dudas.

\section{FASE 3 (RETEST)}

Se otorgaron 40 min para esta fase. Se repitió el mismo procedimiento de la fase 1 para obtener la evaluación postintervención educativa.

Los datos obtenidos se recogieron en una base de datos Excel (variables sociodemográficas y para las MA [test-retest] de antes y después de la intervención educativa). Para el análisis de los datos test-retest antes y después de la intervención educativa, se utilizó el programa NCSS 12 Statistical Software, 2018 (NCSS, LLC. Kaysville, Utah, USA, ncss.com/software/ncss). 


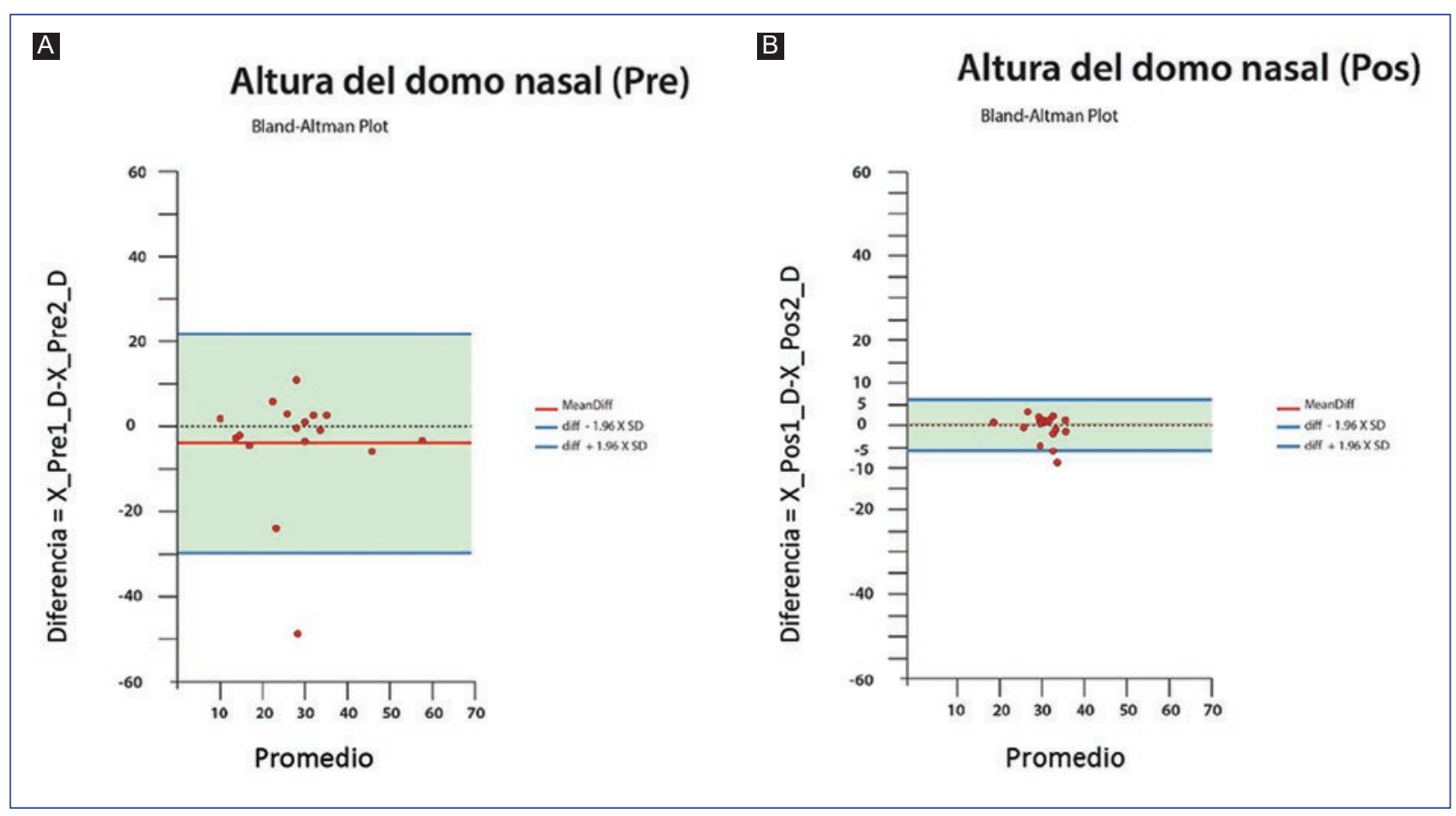

Figura 2. Gráficos Bland-Altman de altura del domo nasal (Pre) (A): la diferencia de medias (MeanDiff, mean difference) fue de $-4.28 \mathrm{~mm}$; la desviación estándar (SD, standard deviation) de 12.96 demuestra que existe una gran dispersión de la forma de medir la altura del domo nasal. El 95\% de los límites de acuerdo se encuentran entre una diferencia de medias de $2.16 \mathrm{~mm}$ a $-10.73 \mathrm{~mm}$. Únicamente se observa un valor extremo. Altura del domo nasal (Pos) (B): después del entrenamiento, la diferencia de medias fue muy cercana a cero $(0.13 \mathrm{~mm})$. El $99 \%$ de la dispersión de los valores se encuentran a menos de 3 SD. El 95\% de los límites de acuerdo se encuentran entre una diferencia de medias de $1.53 \mathrm{~mm}$ a $-1.26 \mathrm{~mm}$. Se observa solamente un valor extremo.

\section{Resultados}

El resultado del análisis del material bibliográfico identificó 13 MA, clasificadas en dimensión vertical, horizontal y ejes, para evaluar la deformidad nasal en pacientes con LPH.

Se registraron 30 asistentes. De estos, se excluyeron 12 , de los cuales ocho no realizaron las mediciones con el instrumento de medición indicado y cuatro no llenaron en su totalidad cualquier cuestionario de la fase 103 . Se incluyeron 18 asistentes que cumplieron con el $100 \%$ de asistencia a la intervención educativa: el $50 \%$ fueron del sexo masculino. El $67 \%$ de los asistentes contaba con la especialidad de ortodoncia, el $12 \%$ eran odontopediatras y el $17 \%$ de la especialidad de cirugía plástica estética y reconstructiva. La media de edad registrada de los asistentes fue 43 años (2765 años). El asistente menos experimentado contaba con 3 años de experiencia, mientras que el asistente con más experiencia tenía 30 años tratando pacientes con LPH.

En la fase 1, se observaron MA con diferencia de medias (DM) diferentes a cero para los casos de la altura del domo nasal (D): DM $-4.28 \mathrm{~mm}$; longitud del ala nasal
(Prn-Ac): DM -2.33 mm; y protrusión de la punta nasal (Sn-Prn): DM -1.87 mm. Estas MA mostraron desviación estándar (DE) de 12.96, 6.25 y 2.96, respectivamente, indicando una dispersión de los datos muy grande.

Después de la fase de intervención educativa, se realizó la medición de la fase 2. Las MA con una diferencia de medias más cercanas a cero fueron el ancho nasal (Sn-Al): DM $0.10 \mathrm{~mm}$; el eje corto de la nariz (Sa sup-Sa inf): DM $-0.11 \mathrm{~mm}$; la altura del domo nasal (D): DM $0.13 \mathrm{~mm}$; y el eje longitudinal de la nariz: DM $-0.13 \mathrm{~mm}$. En comparación con la fase 1, las DE disminuyeron a 1.7, 1.91, 2.8, 1.35 DE, respectivamente.

En la fase 2, se observó que la dispersión de los datos fue menor que en la fase 1; la DE más grande fue la de altura de la base nasal, con 3.7 DE, en comparación con la fase 1, en la cual se registró 6.4 DE. El análisis de los datos de las mediciones previas y posteriores a la presentación de la intervención educativa se muestra en la tabla 1.

En el gráfico Bland-Altman (Fig. 2) se observa la comparación del resultado preintervención y postintervención educativa en la altura del domo nasal. Esta MA mostró una dispersión de datos que confirman una 
Bol Med Hosp Infant Mex. 2020;77(2)

Tabla 1. Diferencias de medias con Bland-Altman de las mediciones antropométricas pre y postintervención educativa

\begin{tabular}{|c|c|c|c|c|c|c|c|c|c|c|}
\hline Medida & DM (Pre) & DE & Límite superior & Límite inferior & CC & DM (Pos) & DE & Límite superior & Límite inferior & CC \\
\hline Longitud de la columnela (Sn-C) & 0.25 & 2.66 & 5.47 & -4.97 & 0.57 & -0.15 & 1.33 & -0.51 & -0.82 & 0.60 \\
\hline Protrusión de la punta nasal (Sn-Prn) & -1.87 & 2.96 & -0.39 & -3.34 & 0.74 & -0.46 & 1.87 & 0.46 & -1.39 & 0.83 \\
\hline Altura de la nariz $(A)$ & -1.17 & 2.05 & -0.14 & -2.19 & 0.40 & -0.37 & 0.97 & 0.10 & -0.85 & 0.39 \\
\hline Distancia vertical del domo & -1.63 & 4.47 & 0.59 & -3.86 & 0.40 & -1.02 & 1.94 & -23 & -2.17 & 0.71 \\
\hline Longitud del ala nasal (Prn-AcR) & -2.33 & 6.25 & 0.77 & -5.44 & 0.63 & 1.07 & 1.07 & 2.85 & -0.71 & 0.75 \\
\hline Altura de la base nasal (C) & 0.19 & 12.54 & 6.49 & -6.04 & 0.37 & 0.47 & 3.75 & 2.33 & -1.39 & 0.76 \\
\hline \multirow[t]{2}{*}{ Altura del domo nasal (D) } & -4.28 & 12.96 & 2.16 & -10.73 & 0.50 & 0.13 & 2.81 & 1.53 & -1.26 & 0.79 \\
\hline & & & & & & & & ¿ & & (Continúa) \\
\hline
\end{tabular}


Tabla 1. Diferencias de medias con Bland-Altman de las mediciones antropométricas pre y postintervención educativa (Continuación)

\begin{tabular}{|c|c|c|c|c|c|c|c|c|c|c|}
\hline Medida & DM (Pre) & DE & Límite superior & Límite inferior & CC & DM (Pos) & DE & Límite superior & Límite inferior & CC \\
\hline Eje longitudinal de la nariz (Nt/NI-Nb/Nm) & -0.60 & 1.99 & 0.38 & -1.59 & 0.62 & -0.13 & 1.35 & 0.54 & -1.80 & 0.84 \\
\hline Eje corto de la nariz (Sa sup-Sa inf) & -0.97 & 2.78 & 0.40 & -2.36 & 0.59 & -0.11 & 1.91 & 0.83 & -1.06 & 0.29 \\
\hline Ancho nasal (Sn-Al) & -1.85 & 4.31 & 0.29 & -4.00 & 0.33 & 0.10 & 1.73 & 0.96 & -0.75 & 0.72 \\
\hline Ancho de la nariz (B) & -1.06 & 3.14 & 0.49 & -2.63 & 0.21 & 0.44 & 0.76 & 0.82 & 0.06 & 0.82 \\
\hline Ancho de la base alar nasal (Sn-Ac) & -0.26 & 3.91 & 1.68 & -2.21 & 0.52 & 0.99 & 1.70 & 1.84 & $\begin{array}{c}0 \\
0.14\end{array}$ & 0.74 \\
\hline Ancho del piso nasal (Sn-Sbal) & 0.36 & 3.23 & 1.24 & -1.97 & 0.56 & -0.31 & 2.03 & 0.59 & -1.31 & 0.59 \\
\hline \multicolumn{11}{|c|}{ 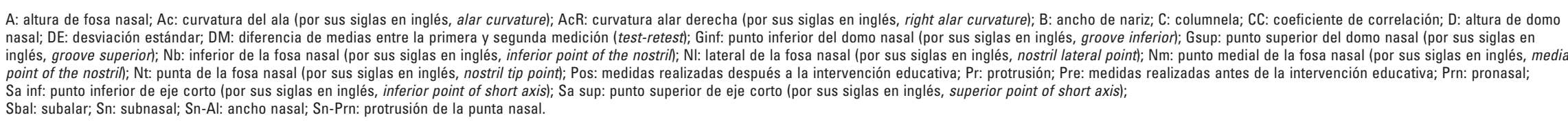 } \\
\hline
\end{tabular}


heterogeneidad en la evaluación de la fase 1. Después de la intervención, se observó una homogeneidad entre los evaluadores en esta MA.

\section{Discusión}

El LPH presenta alteraciones anatómicas, como la deformidad nasal. En 2013, Mani, et al. ${ }^{7}$ observaron que la disminución de la satisfacción en la apariencia nasal se relaciona con una menor calidad de vida y salud mental, razón por la cual se han realizado múltiples esfuerzos para evaluar los resultados de diferentes protocolos de tratamiento en pacientes con LPH. El objetivo de este estudio fue evaluar la efectividad de una intervención educativa aplicada a especialistas de tratamientos de LPH para aumentar la precisión en la evaluación de la simetría nasal con MA.

Fisher, et al. ${ }^{5}$, Henry, et al..$^{6}$ y Ozsoy, et al. ${ }^{10}$ han descrito que la deformidad nasal primaria en pacientes con LPH unilateral se caracteriza por un cartílago alar nasal desplazado lateral, inferior y posterior al lado ipsilateral de la fisura, la punta nasal deprimida y deflexionada hacia el lado no fisurado, la fosa nasal ipsilateral a la fisura está orientada más horizontal que vertical y la columnela es significativamente más corta del lado fisurado y desviada al lado no fisurado. La estandarización de los especialistas en el tratamiento de LPH se enfoca en presentar la ubicación de puntos y MA. Fisher, et al. ${ }^{14}$ han descrito que el tratamiento de LPH es un desafío debido a la compleja alteración de la forma nasal, por lo que los conocimientos sólidos y objetivos de la anatomía de la nariz son primordiales para realizar tratamientos de precisión.

En los estudios realizados por Asher-MacDade, et al. ${ }^{15,16}$ y Al-Ghatam, et al. ${ }^{17}$ se analizó la forma nasal con la escala ordinal de Asher-McDade, que evalúa la estética nasolabial por el método fotográfico. Una de las desventajas de esta escala es la incapacidad de cuantificar la asimetría nasal, además de que la evaluación depende de la experiencia de los especialistas. Este estudio propone la medición de la asimetría nasal con antropometría directa, con el objetivo de presentar resultados cuantitativos para determinar la diferencia de asimetría nasal entre las dos hemicaras. La evaluación con antropometría requiere tanto de personal sometido a pruebas de estandarización como de equipo de precisión calibrado ${ }^{10,18,19}$. Si bien los estudios de evaluación de asimetría nasal con resultados cuantitativos en métodos en 3D han sido validados ${ }^{9,10,20}$, presentan algunas desventajas, como el grado de radiación, alto costo, aparatos sofisticados, poco acceso para trasladar los aparatos de escaneo, calibración de los instrumentos y estandarización del personal de evaluación.

El estudio realizado por Ozsoy, et al. ${ }^{10}$ demostró que el método más confiable de evaluación facial es el de 3D; el segundo método más confiable fue la antropometría directa. Esto refuerza el objetivo de este estudio: estandarizar la evaluación de la simetría nasal con MA directas para lograr un acuerdo confiable en el diagnóstico y plan de tratamiento entre los especialistas de atención de pacientes con LPH. Corona-Rivera, et al. ${ }^{3}$ reportaron que uno de cada 358 recién nacidos vivos presenta LPH en México. Debido a la gran población de pacientes con LPH, la evaluación por 3D representa un método costoso y poco accesible. La antropometría directa, como lo mencionan Ozsoy et al. ${ }^{10}$, Farkas $^{18}$ y Doddi y Eccles ${ }^{21}$, aún es considerada el estándar de oro, ya que presenta ventajas como la facilidad en el manejo y adquisición del instrumento de medición y las evaluaciones a bajo costo, de forma sencilla y rápida.

Los resultados obtenidos antes de la intervención educativa demostraron una heterogeneidad en el conocimiento de las mediciones antropométricas entre los especialistas en el tratamiento de pacientes con LPH. Una de las principales características de la MA es la necesidad de estandarización ${ }^{10}$.

En la primera fase del estudio, cinco de las $13 \mathrm{MA}$ mostraron una DM > 1.5. Esto significa que la magnitud de diferencias entre la primera y segunda medición (test-retest) de la primera fase fue $>1.5 \mathrm{~mm}$. La falta de estandarización entre los especialistas puede conducir a un error en la medición y dar falsos resultados de asimetría nasal. Fourie, et al. ${ }^{22}$ reportaron que una diferencia de $1.5 \mathrm{~mm}$ entre las hemicaras puede ser clínicamente significativa, lo cual destaca la importancia de aumentar el grado de acuerdo en la lectura e interpretación de las MA y evitar errores en la medición.

Durante la fase 2, 11 de las 13 MA disminuyeron la DM. Se observó una $\mathrm{DM} \geq 1 \mathrm{~mm}$ en la longitud del ala nasal y el ancho de la base alar nasal. La curvatura alar (Ac) es un punto antropométrico común en las dos mediciones, definido por Farkas ${ }^{18}$ como el punto más lateral de la inserción del ala nasal en la superficie facial. De acuerdo con la anatomía de pacientes con LPH, esta posición dificulta la localización del punto antropométrico. Sin embargo, la corrección del ancho nasal debe ser uno de los principales objetivos en el cierre de labio y, de acuerdo con Ozsoy et al. ${ }^{10}$ y $\mathrm{Li}$ et al. ${ }^{23}$, el desplazamiento lateroposterior del 
cartílago define el ancho de la fosa nasal; por lo tanto, estos puntos deben evaluarse.

Después a la intervención educativa, la dispersión de las MA disminuyó. Esto indica que las MA no eran entendidas con la misma definición por los especialistas. En la fase 2 se obtuvieron resultados de DE menores que en la fase 1, lo que demuestra que, después de la intervención educativa, se observó una mejor repetibilidad, menor variabilidad y mayor acuerdo en las mediciones. Estos datos de homogeneidad se pueden observar con los gráficos de Bland-Altman ${ }^{24}$ (Fig. 2).

Se concluye que los resultados obtenidos después de la intervención educativa con el manual de procedimientos para la evaluación de la asimetría nasal demuestran que esta es una herramienta útil para estandarizar los conocimientos de MA en los pacientes con LPH.

El acuerdo en la evaluación con MA unifica la forma de medir la asimetría o deformidad nasal, con lo que aumenta la comunicación entre los especialistas de pacientes con LPH para un diagnóstico y un plan de tratamiento confiable. El mayor acuerdo de evaluación entre los especialistas de pacientes con LPH conlleva a una estandarización, que a su vez disminuye el error en la medición y ayuda a tomar decisiones clínicas de tratamiento con métodos confiables.

La medición cuantitativa de la simetría nasal puede cambiar la perspectiva de evaluación y ayudar a crear evidencia basada en los protocolos de tratamiento de los pacientes con LPH.

\section{Responsabilidades éticas}

Protección de personas y animales. Los autores declaran que para esta investigación no se han realizado experimentos en seres humanos ni en animales.

Confidencialidad de los datos. Los autores declaran que en este artículo no aparecen datos de pacientes.

Derecho a la privacidad y consentimiento informado. Los autores declaran que en este artículo no aparecen datos de pacientes. Las fotos faciales tan sólo indican de forma esquemática las medidas antropométricas.

\section{Conflicto de intereses}

Los autores declaran no tener ningún conflicto de intereses.

\section{Financiamiento}

Ninguno.

\section{Agradecimientos}

A Smile Train por las becas de asistencia otorgadas para el XVI Congreso de la Asociación Mexicana de Labio, Paladar Hendido y Anomalías Craneofaciales, A.C.

\section{Bibliografía}

1. Sarmiento K, Valencia S, Gracia G, Hurtado-Villa P, Zarante I. Clinical and epidemiologic description of orofacial clefts in Bogota and Cali, Colombia, 2001-2015. Cleft Palate Craniofac J. 2018;55:517-20.

2. Tanaka SA, Mahabir RC, Jupiter DC, Menezes JM. Updating the epidemiology of cleft lip with or without cleft palate. Plast Reconstr Surg. 2012;129:511e-8.

3. Corona-Rivera JR, Bobadilla-Morales L, Corona-Rivera A, Peña-Padilla C, Olvera-Molina S, Orozco-Martín MA, et al. Prevalence of orofacial clefts and risks for nonsyndromic cleft lip with or without cleft palate in newborns at a university hospital from West Mexico. Congenit Anom (Kyoto). 2018;58:117-23

4. Evans CA. Orthodontic treatment for patients with clefts. Clin Plast Surg. 2004:31:271-90

5. Fisher DM, Tse R, Marcus JR. Objective measurements for grading the primary unilateral cleft lip nasal deformity. Plast Reconstr Surg. 2008;122:874-80.

6. Henry C, Samson T, Mackay D. Evidence-based medicine: the cleft lip nasal deformity. Plast Reconstr Surg. 2014;133:1276-88.

7. Mani M, Reiser E, Andlin-Sobocki A, Skoog V, Holmström M. Factors related to quality of life and satisfaction with nasal appearance in patients treated for unilateral cleft lip and palate. Cleft Palate Craniofac J. 2013; 50:432-9.

8. Puente EJ, Ortiz M, Garay F. Análisis fotogramétrico de las alteraciones en estructuras nasales cartilaginosas y de tejidos blandos en los pacientes con labio y paladar hendido unilateral no sindromático previamente operados [tesis de especialidad]. Ciudad Universitaria, México: Universidad Nacional Autónoma de México; 2011.

9. Hood CA, Hosey MT, Bock M, White J, Ray A, Ayoub AF. Facial characterization of infants with cleft lip and palate using a three-dimensional capture technique. Cleft Palate Craniofac J. 2004;41:27-35

10. Ozsoy U, Demirel BM, Yildirim FB, Tosun O, Sarikcioglu L. Method selection in craniofacial measurements: advantages and disadvantages of 3D digitization of 3D digitization method. J Craniomaxillofac Surg. 2009;37:285-90.

11. Wong JY, Oh AK, Ohta E, Hunt AT, Rogers GF, Mulliken JB, et al. Validity and reliability of craniofacial anthropometric measurement of 3D digital photogrammetric images. Cleft Palate Craniofac J. 2008;45:232-9.

12. Szklo M, Nieto FJ. Aseguramiento y Control de la Calidad. En: Szklo M, Nieto FJ. Epidemiología intermedia, conceptos y aplicaciones. Madrid: Editoral Diaz de Santos; 2003. pp. 297-315.

13. Argimon JM, Jimenez J. Medición de Variables. En: Argimon Pallás JM, Jiménez Villa J. Métodos de investigación clínica y epidemiológica. Madrid: Elsevier; 2004. pp. 168-75.

14. Fisher DM, Fisher DM, Marcus JR. Correction of the cleft nasal deformity: from infancy to maturity. Clin Plast Surg. 2014;41:283-99.

15. Asher-McDade C, Roberts C, Shaw WC, Gallager C. Development of a method for rating nasolabial appearance in patients with clefts of the lip and palate. Cleft Palate Craniofac J. 1991;28:385-90.

16. Asher-MacDade C, Brattström V, Dahl E, McWilliam J, Mølsted K, Plint DA, et al. A six-center international study of treatment outcome in patients with clefts of the lip and palate. Part 4. Assessment of nasolabial appearance. Cleft Palate Craniofac J. 1992;29:409-12.

17. Al-Ghatam R, Jones TE, Ireland AJ, Atack NE, Chawla O, Deacon S, et al. Structural outcomes in the Cleft Care UK study. Part 2. Dento-facial outcomes. Orthod Craniofac Res. 2015;18:14-24.

18. Farkas LG. Accuracy of anthropometric measurements: past, present and future. Cleft Palate Craniofac J. 1996;33;10-8.

19. Doddi NM, Eccles R. The role of anthropometric measurements in nasal surgery and research: a systematic review. Clin Otolaryngol. 2010;35:277-83.

20. Nkenke E, Lehner B, Kramer M, Haeusler G, Benz S, Schuster M, et al. Determination of facial symmetry in unilateral cleft lip and palate patients from three-dimensional data: technical report and assessment of measurement errors. Cleft Palate Craniofac J. 2006:43:129-37.

21. Doddi NM, Eccles R. The role of anthropometric measurements in nasal surgery and research: a systematic review. Clin Otolaryngol. 2010;35:277-83.

22. Fourie Z, Damastra J, Gerrits PO, Ren Y. Evaluation of anthropometric accuracy and reliability using different three dimensional scanning systems. Forensic Sci Int. 2011;207:127-34.

23. Li G, Wei J, Wang X, Wu G, Ma D, Wang B. Three dimensional facial anthropometry of unilateral cleft lip infants with a structured light scanning system. J Plast Reconstr Aesthet Surg. 2013;66:1109-16.

24. Giovarina D. Understanding Bland Altman analysis. Biochem Med (Zagreb). 2015;25:141-51. 